The Versatile Transceiver: towards production readiness

This content has been downloaded from IOPscience. Please scroll down to see the full text. 2013 JINST 8 C03004

(http://iopscience.iop.org/1748-0221/8/03/C03004)

View the table of contents for this issue, or go to the journal homepage for more

Download details:

IP Address: 137.138.125.163

This content was downloaded on 08/07/2014 at 08:35

Please note that terms and conditions apply. 
Topical Workshop on Electronics for Particle Physics 2012, 17-21 SEPTEMBER 2012, OXFORD, U.K.

\title{
The Versatile Transceiver: towards production readiness
}

\author{
C. Soós, ${ }^{1}$ M. Barros Marin, S. Détraz, L. Olanterä, C. Sigaud, S. Storey, J. Troska, \\ F. Vasey and P. Vichoudis \\ CERN, \\ 1211 Geneva 23, Switzerland \\ E-mail: Csaba.Soos@cern.ch
}

ABSTRACT: Detectors involved in the upgrade programme of the LHC will need high-speed optical links to transfer readout and control data. The link front-end will be based on a radiation tolerant opto-electronic module, the Versatile Transceiver (VTRx), developed under the Versatile Link project. In this contribution we present a test system and protocol to be used to verify the compliance of the VTRx modules to the specifications, and a Versatile Link demonstrator based on the VTRx and the Gigabit Link Interface Board. Finally, we introduce the Small Footprint VTRx which is being designed for the CMS Tracker upgrade.

KEYWORDS: Optical detector readout concepts; Radiation-hard electronics; Front-end electronics for detector readout

\footnotetext{
${ }^{1}$ Corresponding author.
} 


\section{Contents}

1 Introduction 1

2 Towards production $\quad 2$

2.1 Prototypes 2

2.2 Test results 2

3 Procurement strategy 4

4 Quality assurance $\quad 5$

5 System demonstrator 6

6 Small footprint Versatile Transceiver $\quad 7$

$\begin{array}{llr}7 & \text { Summary } & 8\end{array}$

\section{Introduction}

The high data rate and the presence of increased radiation at the upgraded LHC experiments call for high-speed optical links based on radiation tolerant front-end interfaces. In addition, merging the detector data readout with the timing and control distribution on the same optical link requires bi-directional functionality. The Versatile Transceiver (VTRx) developed in the framework of the Versatile Link project $[1,2]$ addresses these needs. Single-mode and multi-mode transceiver variants are available for readout systems using different fibre plants. For detector systems where the number of readout channels exceeds that of control channels, the Versatile Twin Transmitter (VTTx) can be deployed to increase the readout density. The VTRx and VTTx operate at $4.8 \mathrm{~Gb} / \mathrm{s}$ data rate and use radiation hard ASICs sourced from the GigaBit Transceiver (GBT) project [3]. The paper will summarize these variants, their main characteristics, and indicate the production quantities to be used by early adopters.

Following the feasibility study and an extensive prototyping phase, the Versatile Link project moved into a pre-production readiness phase at the end of 2011. Packaged VTRx and VTTx modules are being produced in small series to verify manufacturability and to provide samples to early adopters. The fully functional prototypes supporting either single-mode or multi-mode operation are built using the GBT chipset ASICs $[4,5]$ and commercial off-the-shelf components that were previously qualified for use in radiation environments [6, 7]. During the procurement phase, transceiver components will be purchased from different vendors and transceiver modules will be produced in larger quantities. Section 4 describes an easy-to-execute test protocol and a dedicated VTRx/VTTx test system which will be used to evaluate the components and to screen 
Table 1. Available VTRx and VTTx variants.

\begin{tabular}{|c|c|c|c|}
\hline Variant & Laser Driver & TOSA type & ROSA type \\
\hline Single-mode VTRx & GBLD & $1310 \mathrm{~nm}$ EEL & $60 \mu \mathrm{m} \mathrm{InGaAs}$ \\
\hline Multi-mode VTRx & GBLD & $850 \mathrm{~nm}$ VCSEL & $70 \mu \mathrm{m} \mathrm{GaAs}$ \\
\hline Multi-mode VTTx & GBLD & $850 \mathrm{~nm}$ VCSEL & n.a. \\
\hline Multi-mode VTTx & COTS LDD & $850 \mathrm{~nm}$ VCSEL & n.a. \\
\hline
\end{tabular}

problems during manufacturing, and to verify the module compliance with the Versatile Link specifications [8]. In addition, we have implemented a Versatile Link demonstrator using the Gigabit Link Interface Board (GLIB) [9] which allows the user to get started using the VTRx and to evaluate the performance of the Versatile Link. The architecture of the demonstrator and some tests results will be presented in section 5 .

Finally, section 6 will introduce a new flavour of the transceiver which is proposed for the Phase II upgrade of the CMS Tracker system. The Small Footprint VTRx (SF-VTRx) will allow a more compact placement on the detector module by reducing the real-estate used for electrical and optical connections.

\section{Towards production}

\subsection{Prototypes}

Four different VTRx and VTTx variants are available for users that are ready to integrate the modules into their upgraded readout systems. The variants and their main components are summarized in table 1. The transmitter path of VTRx modules consists of the radiation-tolerant GigaBit Laser Driver (GBLD) and a commercial Transmitter Optical Sub-Assembly (TOSA). The single-mode version is based on an Edge Emitter Laser (EEL) operating at $1310 \mathrm{~nm}$, while the multi-mode variant uses a Vertical Cavity Surface Emitting Laser (VCSEL) operating at $850 \mathrm{~nm}$. On the receiver side the Receiver Optical Sub-Assembly (ROSA) includes the radiation-tolerant GigaBit TransImpedance and limiting Amplifier (GBTIA) and either a $60 \mu \mathrm{m}$ InGaAs or a $70 \mu \mathrm{m}$ GaAs commercial PIN photodiode to support single-, or multi-mode operations respectively. There are two multi-mode VTTx variants available: the radiation hard version uses the GBLD while the radiation soft version is based on a commercial off-the-shelf laser diode driver (COTS LDD). In both cases the modules are assembled with VCSELs operating at $850 \mathrm{~nm}$.

Fully functional prototypes of the above mentioned variants have been fabricated in small quantities. The prototypes were tested in the laboratory and the results are discussed in section 2.2. The picture of a multi-mode VTRx prototype is shown in figure 1.

\subsection{Test results}

The performance of the prototypes listed in table 1 was evaluated by measuring the transmit optical eye diagram of the VTRx and VTTx modules, as well as the receiver electrical eye and the receiver sensitivity of the VTRx modules. The measured parameters have been compared with the specifications. 


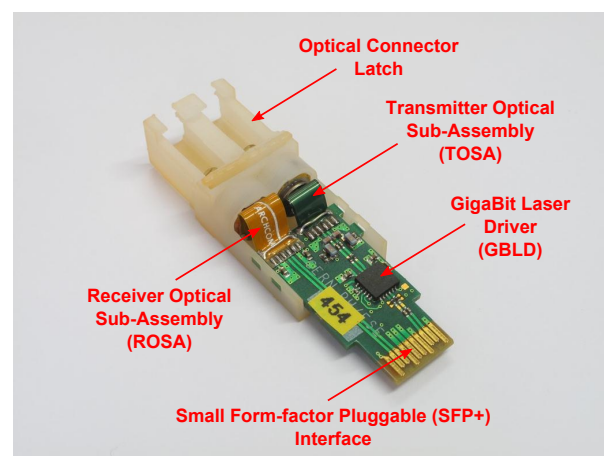

Figure 1. Picture of a multi-mode VTRx variant showing the main components of the transceiver module.

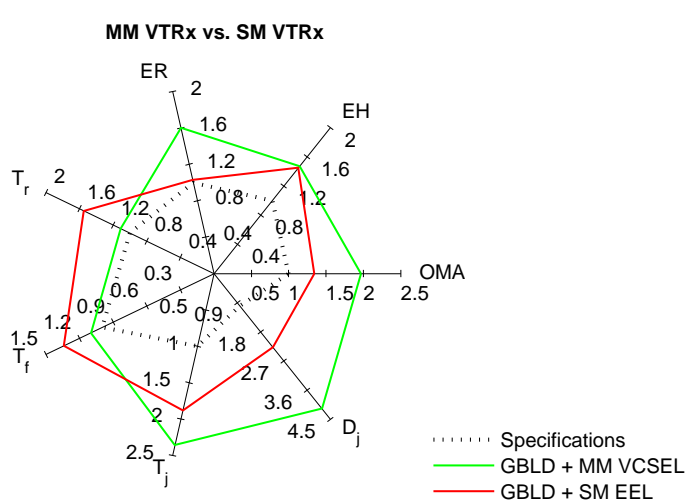

(a)

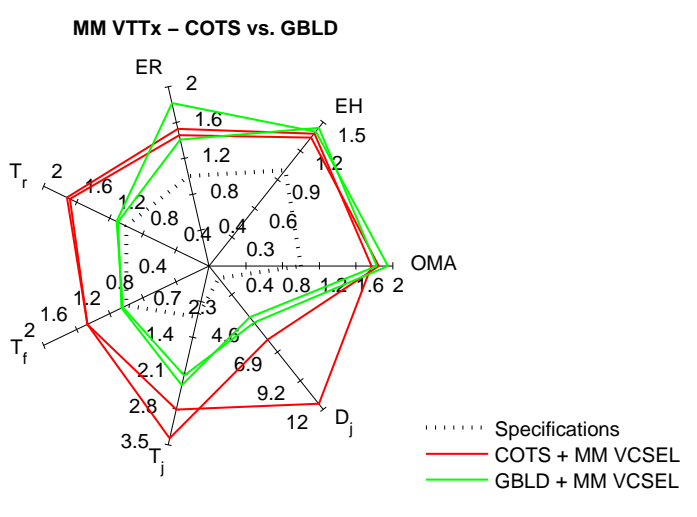

(b)

Figure 2. Radar plots showing normalized eye diagram parameters for single-mode and multi-mode VTRx variants (a) as well as for multi-mode VTTx variants based on a commercial laser driver or the GBLD (b).

The eye diagram parameters presented hereafter have been normalized using the target values listed in table 2. Following normalization parameters that do not meet the specifications are represented by numbers smaller than 1 . The radar plot in figure 2.a shows the results measured using the multi-mode and single-mode VTRx variants. The plot confirms that specifications, represented by a dotted line, are met in both cases. Figure 2.b summarizes the parameters obtained by measuring COTS-based and GBLD-based VTTx prototypes. The better timing and jitter characteristics of the commercial laser driver can be explained by the fact that the device is designed for $10 \mathrm{G}$ applications.

The receiver sensitivity of the single-mode and multi-mode transceiver prototypes have been measured using a bit error rate (BER) tester. The BER curves and the corresponding sensitivity limits (vertical dashed lines) are shown in figure 3. According to the Versatile Link specifications [8] these limits are defined as $-15.4 \mathrm{dBm}$ and $-13.1 \mathrm{dBm}$ for the single-mode and multi-mode cases, respectively. Results show that the specifications have been met in both cases. 
Table 2. Target eye diagram parameters.

\begin{tabular}{|l|l|l|}
\hline Parameter & Target & Unit \\
\hline Optical Modulation Amplitude (OMA) & 300 & $\mu \mathrm{W}$ \\
\hline Eye Height (EH) & $0.6^{*} \mathrm{OMA}$ & $\mu \mathrm{W}$ \\
\hline Extinction Ratio (ER) & 3 & $\mathrm{~dB}$ \\
\hline Rise time $\left(t_{r}\right)$ & 70 & $\mathrm{ps}$ \\
\hline Fall time $\left(t_{f}\right)$ & 70 & $\mathrm{ps}$ \\
\hline Total jitter $\left(T_{j}\right)$ at BER $=10^{-12}$ & 52 & $\mathrm{ps}$ \\
\hline Deterministic jitter $\left(D_{j}\right)$ & 26 & $\mathrm{ps}$ \\
\hline
\end{tabular}

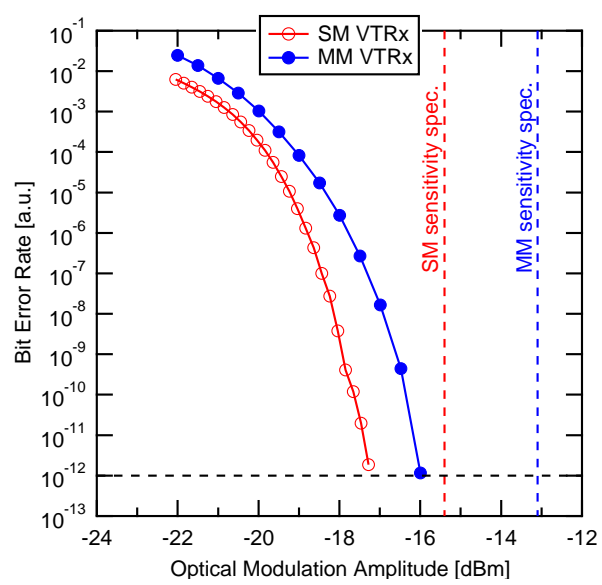

Figure 3. Bit error rate curves measured using a single-mode and a multi-mode VTRx.

\section{Procurement strategy}

A purchasing strategy is being defined to supply optical link components to the following users identified to date: ATLAS Small Wheel, ATLAS Liquid Argon Calorimeter, LHCb, and CMS Hadron Calorimeter upgrade. Depending on quantity of components to be purchased and the amount of CERN funds used to pay for the supply the following procedures are being investigated:

1. Market Survey, Invitation to Tender and Contract Placement;

2. Price Enquiry and Contract Placement;

3. Single-source purchase orders.

Procedure 1 will be used to procure the large number of multi-mode TOSA devices. For purchasing moulded multi-mode variant of the plastic optical connector latch and to manufacture VTRx and VTTx modules we will follow procedure 2. Finally, procedure 3 will be used for purchasing ROSA devices and the machined version of the single-mode optical latch, as well as to order single-mode TOSA devices which are known to meet our technical specifications and are significantly more rad-hard than other candidate components that we tested. The procurement will start in 2013 allowing enough time for purchasing all components required to manufacture the modules in 2014. 


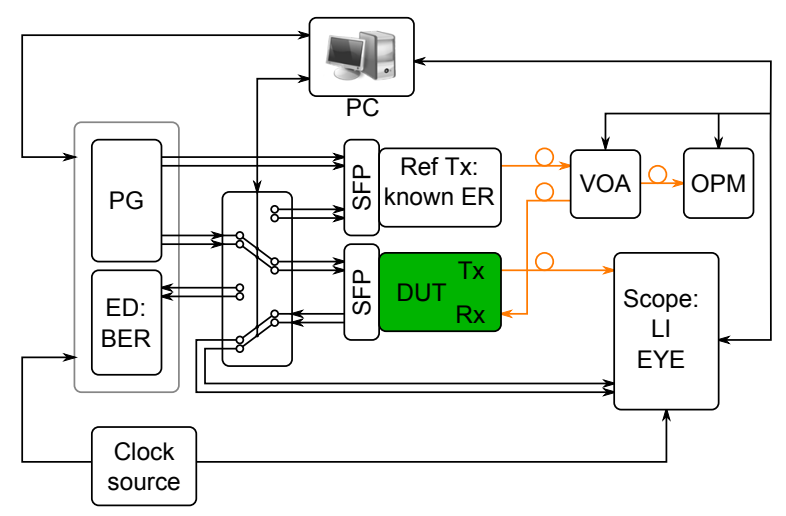

Figure 4. Experimental setup proposed for production testing of VTRx modules.

\section{Quality assurance}

Quality Assurance (QA) has been implemented via various measures taken throughout the different phases of the Versatile Link project. During the feasibility study, all candidate components of the VTRx and VTTx were thoroughly tested as part of the qualification process. Functional and environmental tests have been conducted in the lab $[10,11]$ as well as in irradiation facilities [6, 7]. Similar tests will be carried out during the procurement procedure (see section 3) to evaluate the performance of the devices proposed by the manufacturers. In addition, acceptance tests will be done to control the quality of the delivered parts. However, since these time consuming tests cannot be done for each manufactured module a different procedure is being defined for production testing.

The purpose of the production test is to verify that the manufactured modules function properly and that the measured parameters are within the limits set by the specifications. Unlike during full characterization, in production testing the measurement time must be optimized to allow testing of several thousand modules within a reasonable time. The test setup should support quasi-automated testing and the role of the human operator should be limited to the replacement of the device under test.

The test procedure consists of measuring the light-current (LI) characteristics, the transmit optical eye, the receive electrical eye and the bit error rate (BER). The proposed test setup is shown in figure 4. The test pattern is generated using a Pattern Generator (PG) which is connected either to the reference transmitter or to the Device Under Test (DUT) through a high-speed analogue switch, which allows the rerouting of the signals for different measurement configurations. The optical output of the DUT is connected to an oscilloscope which will measure the LI curve and the TX optical eye. The optical signal from the reference transmitter is fed through a Variable Optical Attenuator (VOA) before reaching the receiver side of the DUT. The average optical power of the attenuated signal is measured using an Optical Power Meter (OPM) attached to the monitoring output of the VOA. The electrical output from the DUT receiver is going through the analogue switch, which can connect the signal either to the error detector (ED) to measure BER or to the oscilloscope to capture the received electrical eye.

Based on the requirements we have built a test system using bench-top instruments. For multiplexing multi-gigabit signals we identified a high-bandwidth passive switch. We ordered an eval- 


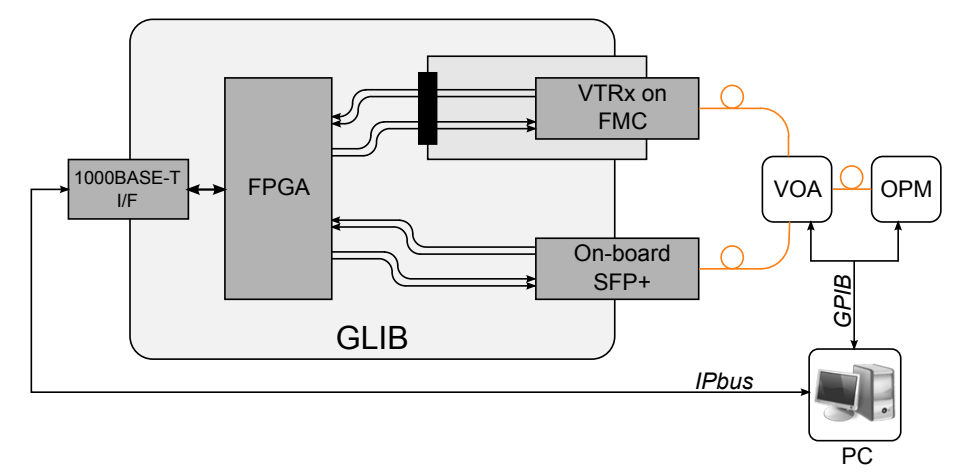

Figure 5. GLIB-based Versatile Link system demonstrator.

uation board from the chip vendor, but it did not arrive in time and we could not evaluate its performance before submitting the paper. However, we were able to use the system to optimize the test procedure and to develop the necessary software components, as well as to test the prototypes mentioned in section 2.1.

\section{System demonstrator}

As presented in section 2.1, fully functional VTRx and VTTx prototypes are available for the interested users. To start the system-level evaluation users will need to build their own test systems. Moreover, in many cases this evaluation shall take place well before an appropriate front-end prototype hosting the VTRx/VTTx becomes available. The purpose of the system demonstrator is to provide a verified turn-key solution that speeds up the integration with the Versatile Link.

The architecture has been derived from the one shown in figure 4. However, instead of an expensive and less portable pattern generator/error checker instrument the demonstrator is built around the Gigabit Link Interface Boad (GLIB) which is an evaluation board developed at CERN. The GLIB is an extensible platform that enables easy integration with VTRx/VTTx modules as well as with commercial transceivers. The board features a large Field Programmable Gate Array (FPGA) device which can be loaded with a firmware that implements the functions required to operate the system. The demonstrator implements a multi-channel BER tester which supports various test configurations using commercial transceivers and VTRx modules.

Figure 5 shows a typical configuration which can be used to measure the system BER. The VTRx is attached to the GLIB using an FMC-compatible mezzanine adapter. The optical signal from the VTRx is fed through a Variable Optical Attenuator (VOA) and then looped back to the commercial SFP+ transceiver integrated on-board the GLIB. The average power of the attenuated signal, required to calculate the receiver sensitivity, is measured using an Optical Power Meter (OPM). The instruments and the GLIB are controlled using LabVIEW running on a computer. The data generation and the error detection are implemented in the FPGA firmware. The GLIB can encode/decode the bit stream using the GBT protocol [12], thus allowing to measure the systemlevel BER. 


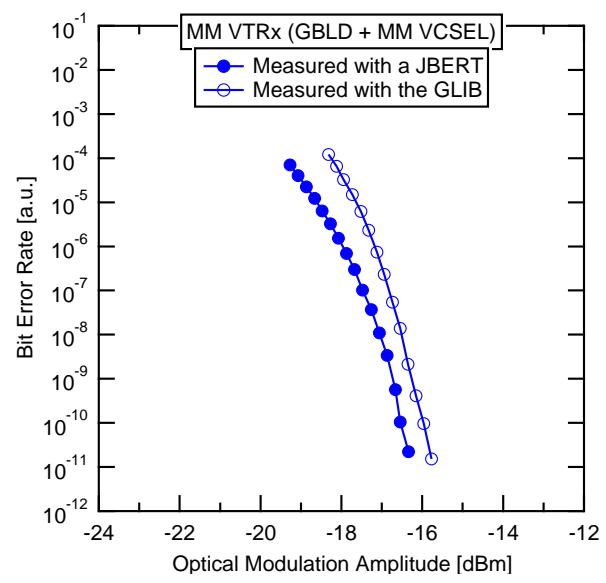

Figure 6. Bit error rate curves of a multi-mode VTRx measured with a dedicated BER tester or the GLIBbased demonstrator.

We built the system using the components described before and we tested some VTRx prototypes with commercial SFP+ transceivers as a reference transmitter. We compared the results with those measured using the instrument-based system presented in section 4 . The two BER curves shown in figure 6 have been measured using the same multi-mode VTRx and commercial transceiver pair and either a dedicated pattern generator/checker (JBERT) or the GLIB-based demonstrator (without error correction). The results reveal that the JBERT has slightly superior performance, but the penalty associated with the usage of the GLIB is only $0.5 \mathrm{~dB}$.

\section{Small footprint Versatile Transceiver}

The future front-end hybrid of the CMS Tracker detector, to be replaced during the LHC Phase-II upgrade, will require a low-profile optical interface like in many other experiments. However, the current version of the VTRx does not fit in the envelope allocated for the link interface. In addition, the $45 \times 15 \times 8 \mathrm{~mm}^{3}$ volume will have to accommodate the future version of the GBT serializer chip. In case of an SFP-like module, such as the VTRx, the PCB area under the transceiver cannot be used. Eliminating the connector and integrating the optical components on the front-end could reduce the footprint, but the advantages of the pluggable module concept would be lost as well.

To reduce the footprint of the transceiver and to allow tighter integration with the serializer both the electrical and optical connections need to be modified as illustrated by the CAD drawing shown in figure 7.a. The surface-mount electrical connector would allow the placement of the serializer chip (GBTX) on motherboard in the area under the transceiver. On the optical interface, the standard LC connector should be replaced by a custom connector, which will be held together with the optical components (TOSA and ROSA) by using a modified plastic latch or a metal clip. Figure 7.b shows the first version of the Small Footprint Versatile Transceiver (SF-VTRx) and one of the VTRx prototypes photographed side-by-side. The picture allows to compare the size of the two objects and it clearly demonstrates how the above mentioned modifications could enable a more compact optical interface for the front-end hybrid. 


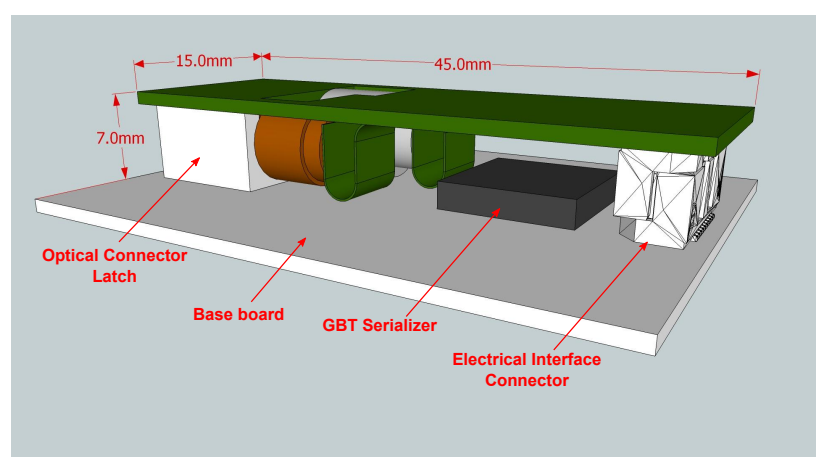

(a)

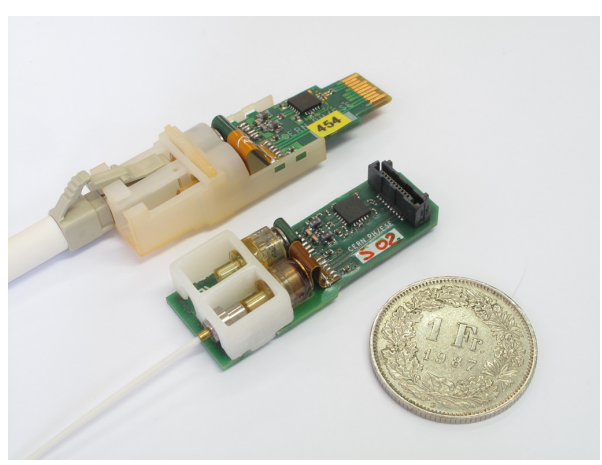

(b)

Figure 7. CAD drawing of the SF-VTRx (a) and a picture of the first prototype (b).

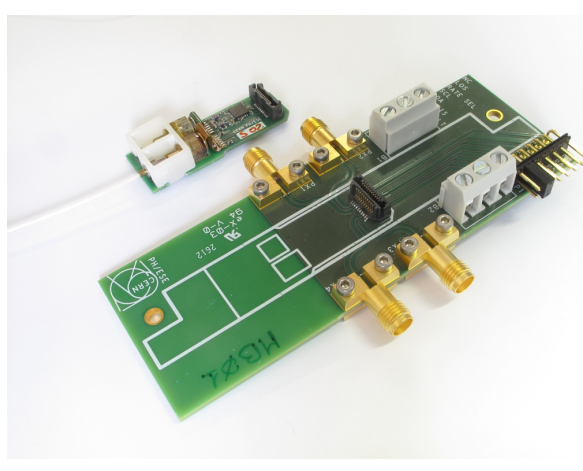

(a)

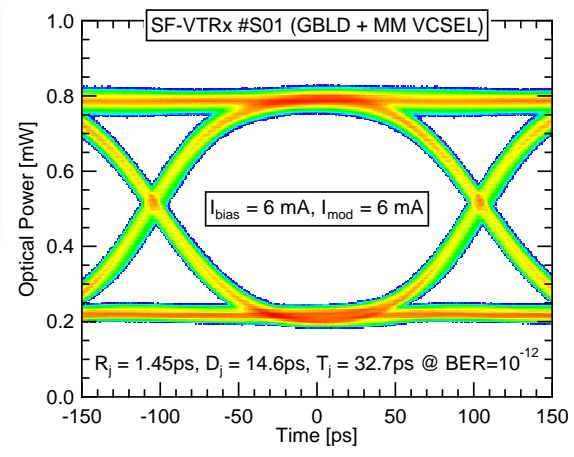

(b)

Figure 8. Picture of the test board used for measuring the SF-VTRx prototypes (a) and the transmit optical eye produced by one of the prototypes (b).

We have assembled two SF-VTRx prototypes using multi-mode VCSELs operating at $850 \mathrm{~nm}$, radiation hard GBLD chips and multi-mode ROSA devices based on the radiation hard GBTIA and $70 \mu \mathrm{m}$ GaAs photodiodes. The performance of the prototypes has been tested using a custom designed board shown in figure 8.a. The transmit optical eye shown in figure 8.b has been measured using the default laser driver settings. The extracted eye diagram parameters confirm that the prototypes meet the Versatile Link specifications.

\section{Summary}

The Versatile Link project has moved to the pre-production readiness phase. Fully functional VTRx and VTTx prototypes have been produced in small quantities and test results confirm that these prototypes meet the Versatile Link specifications. The first Versatile Link users have been identified and a tentative procurement strategy has been defined. A test setup supporting quasi-automated production testing is being implemented. A system demonstrator based on the Gigabit Link Interface Board is available for users wishing to evaluate the performance of the Versatile Link and the GBT protocol. The concept of the Small Footprint Versatile Transceiver has been introduced, which is offering a low-profile, radiation-hard optical interface for the more long-term Phase-II upgrade of the CMS Tracker detector. 


\section{Acknowledgments}

The authors would like to graciously acknowledge the support of the EU-funded 7th Framework Marie-Curie ACEOLE programme.

\section{References}

[1] L. Amaral et al., The Versatile Link, A Common Project For Super-LHC, 2009 JINST 4 P12003.

[2] F. Vasey et al., The Versatile Link common project: feasibility report, 2012 JINST 7 C01075.

[3] P. Moreira et al., The GBT Project, in Proc. of TWEPP 2009 Topical Workshop on Electronics for Particle Physics, September, 21-25, 2009 Paris, France, CERN-2009-006.

[4] G. Mazza et al., A 5Gb/s Radiation Tolerant Laser Driver in $130 \mathrm{~nm}$ CMOS technology, 2012 JINST $7 \mathrm{C} 01052$

[5] M. Menouni et al., The GBTIA, a 5 Gbit/s radiation-hard optical receiver for the SLHC upgrades, in Proc. TWEPP 2009 Topical Workshop on Electronics for Particle Physics, September, 21-25, 2009 Paris, France, CERN-2009-006.

[6] J.Troska et al., Radiation damage studies of lasers and photodiodes for use in multi-Gb/s optical data links, IEEE Trans. Nucl. Sci. 58 (2011) 3103.

[7] J. Troska et al., Single-Event Upset Testing of the Versatile Transceiver, 2011 JINST 6 C11026.

[8] J. Troska et al., Versatile Link Specification Part 2.1 Front-end Versatile Transceiver, EDMS-1140665-1.

[9] P. Vichoudis et al., The Gigabit Link Interface Board (GLIB), a flexible system for the evaluation and use of GBT-based optical links, 2010 JINST 5 C11007.

[10] C. Soós et al., Versatile transceiver development status, 2012 JINST 7 C01094

[11] L. Olanterä et al., Temperature Characterization of Versatile Transceivers, submitted to the proceedings of TWEPP 2011 Topical Workshop on Electronics for Particle Physics, September, 17-21, 2012 Oxford, UK.

[12] G. Papotti et al., An Error-Correcting Line Code for a HEP Rad-Hard Multi-GigaBit Optical Link, in proceedings of the 12th Workshop on Electronics for LHC and Future Experiments, September, 25-29, 2006 Valencia, Spain, CERN-LHCC-2007-006. 